\title{
FACTORES A CONSIDERAR EN LA REGENERACIÓN NATURAL DEL BOSQUE TROPICAL SECO EN NICARAGUA
}

\section{FACTORS TO CONSIDER IN THE NATURAL REGENERATION OF TROPICAL DRY FOREST IN NICARAGUA}

\section{Gonzalez-Rivas Benigno, Castro-Marín Guillermo}

Facultad de Recursos Naturales y del Ambiente, Universidad Nacional Agraria, Apartado postal 453, Managua, Nicaragua. E-mail: Benigno.Gonzalez@una.edu.ni, Guillermo.Castro@una.edu.ni

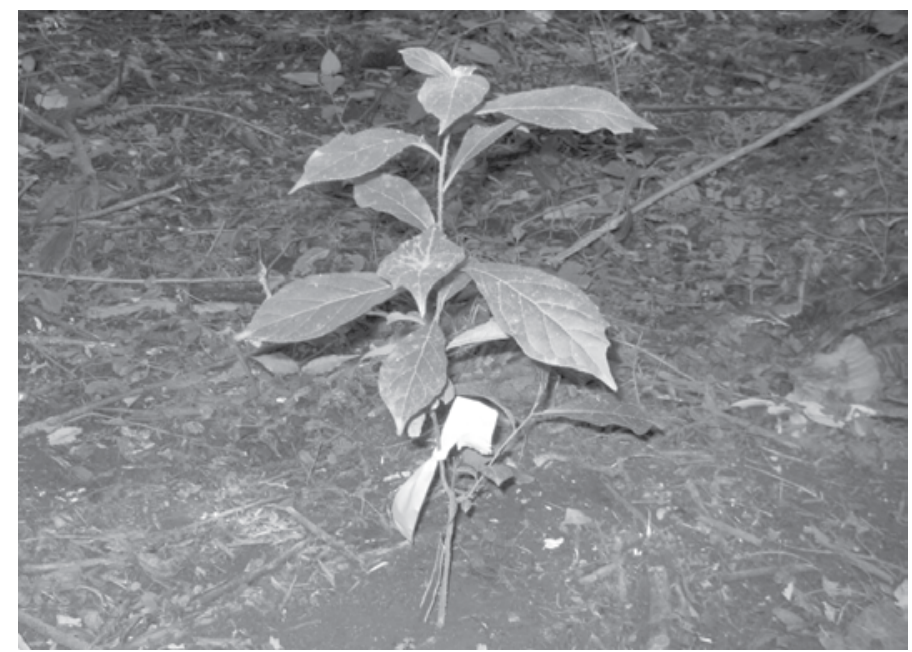

\section{RESUMEN}

En el presente estudio se analizó el banco de semillas del suelo en un ecosistema de bosque seco secundario en Nandarola, Nandaime, departamento de Granada. La investigación tuvo por objetivos estimar composición florística y densidad de semillas presentes en el banco de semillas de suelo. Se recolectaron muestras de suelo que incluían la capa litter y tres capas de suelo mineral sucesivas (de 0-3, 3-6 y 6-9 cms). El número total de especies presente en el banco de semillas del suelo fue de 33 especies, distribuidas en 23 familias. El número total de semillas presente fue 240 semillas por metro cuadrado. Tambien se hizo otro estudio sobre la regeneración natural de tres especies: Lysiloma divaricatum (Jacq.), Tabebuia ochracea (Cham.) and Lonchocarpus minimiflorus (Donn. Sm.) en un periodo de tres años en la Reserva Natural de Chacocente. Los resultados obtenidos señalan que la densidad de las especies varía significativamente durante el periodo de estudio. La regeneración natural por si sola no es suficiente para mantener el número adecuado de plántulas, por lo tanto se deben de tomar medidas para incrementar la regeneración de las especies.

Palabras clave: América Central, mortalidad, regeneración natural, bosque seco

\section{ABSTRACT}

The present study the soil seed bank was analyzed in an ecosystem of secondary dry forest in Nandarola, Nandaime, department of Granada. The main objectives were to determine floristic composition, and density of the soil seeds bank. The soil sampling was systematic and included the litter layer and three mineral layers successive $(0-3,3-6$ and 6-9 $\mathrm{cms})$. The number of species found was 33 species and 23 families. Another study was carried out in dry forest of Chacocente Wildlife Reserve (11 $36^{\prime}-11^{\circ} 30^{\prime} \mathrm{N}$ and $86^{\circ} 08^{\prime}-86^{\circ} 15^{\prime} \mathrm{W}$ ) located on the Pacific coast in the department of Carazo. The study includes the natural regeneration of three forest species: Lysiloma divaricatum (Jacq.), Tabebuia ochracea (Cham.) and Lonchocarpus minimiflorus (Donn. Sm.) . The results show that the density of naturally regenerated individuals varied significantly among species, as well as over time. We concluded that natural regeneration alone is not sufficient to maintain the desired number of stocks of these species, and an immediate restoration measure should be taken to assist the natural regeneration process.

Key words: Central America, mortality, natural regeneration, dry forest 
$\mathrm{E}$ xisten evidencias que indican que el bosque tropical seco en América Central ha sido convertido en otros usos tal como agricultura y pastoreo (Brown y Lago 1990). El bosque tropical seco que aún queda equivale a menos del $2 \%$ del bosque original. El bosque seco en muchas áreas de América central se presenta como fragmentos y se considera como un mosaico de formaciones de bosque secundario ( Kalacska 2004)

Nicaragua tiene 100000 ha de bosque seco localizado principalmente en el Pacifico del país. Por lo tanto, existe bastante interés de proteger y rehabilitar el bosque remanente.

El manejo de la regeneración natural es un método barato para rehabilitar este tipo de ecosistema, basado en el banco de semillas del suelo y la existencia de arboles padres que pueden servir como suplidores de semillas, por lo tanto es necesario evaluar y cuantificar el banco de semillas y la regeneración natural para determinar si es necesario aplicar otro tipo de intervenciones en el bosque para acelerar el proceso de regeneración natural en el bosque (Teketay 1997).

La restauración de un bosque secundario en un campo abandonado depende de la disponibilidad de semillas (Zimmerman et al. 2000). La disponibilidad de semillas se puede caracterizar por la presencia, ganancia y pérdida de semillas viables. La presencia de semillas en el banco de semillas del suelo puede ser alterado por la duración, frecuencia y la intensidad de los disturbios en el bosque (Teketay 1997; Tekle y Bekele 2000).

\section{MATERIALES Y MÉTODOS}

Sitios de estudio. Los sitios de estudio fueron en La Chipopa, Nandaime, donde se hizo el estudio de banco de semillas del suelo y el Refugio de Vida Silvestre de Chacocente donde se hizo el estudio de la regeneración natural (Figura 1)

La comunidad de La Chipopa se ubica a casi 12 kilómetros al suroeste de la ciudad de Nandaime, departamento de Granada, al oeste del gran lago de Nicaragua (Figura 1).La vegetación presentaespecies como: Acacia collinsii (cornizuelo), Gliricidia sepium (madero negro), Erythrina fusca (helequeme), entre otras; aunque también el sotobosque es dominado por especies herbáceas y lianas. Cuenta con un área de 3.83 hectáreas, localizada en las coordenadas 11o 42' $31^{\prime \prime}$ Norte y $86^{\circ} 05^{\prime} 08^{\prime \prime}$ Oeste, siendo su altitud promedio de $151 \mathrm{msnm}$.

El Refugio de Vida Silvestre de Chacocente está localizado en la costa del Pacifico de Nicaragua específicamente en el Departamento de Carazo (Figura 1). El área total de la reserva es de 4645 ha. De acuerdo a la clasificación de Holdridge el bosque es clasificado como Bosque seco tropical.
Los bosques tropicales secos de Meso América contienen una alta riqueza estructural y funcional (Murphy \& Lugo 1986), proveen numerosos servicios ambientales, y constituyen un modelo para la restauración ecológica y el estudio de la regeneración de los ecosistemas tropicales (Janzen 2000). Estas zonas de vida han sido las predilectas para el desarrollo de importantes asentamientos huma nos a través del tiempo, lo que ha causado enormes perturbaciones ecológicas; los bosques tropicales secos son actualmente un mosaico de fragmentos de bosques sucesionales secundarios (Kramer 1997).

El éxito de los esfuerzos para restaurarlos el bosque seco tropical dependerá en gran medida del conocimiento de los procesos de regeneración natural y la dinámica del banco de semillas del suelo (Bazzaz \& Pickett 1980, Mulkey et al. 1996).

Este estudio persigue estudiar la abundancia y frecuencia de la regeneración natural de algunas especies forestales en un lapso de dos años. Además, conocer la composición y riqueza de especies presentes en el banco de semillas del suelo y su densidad.

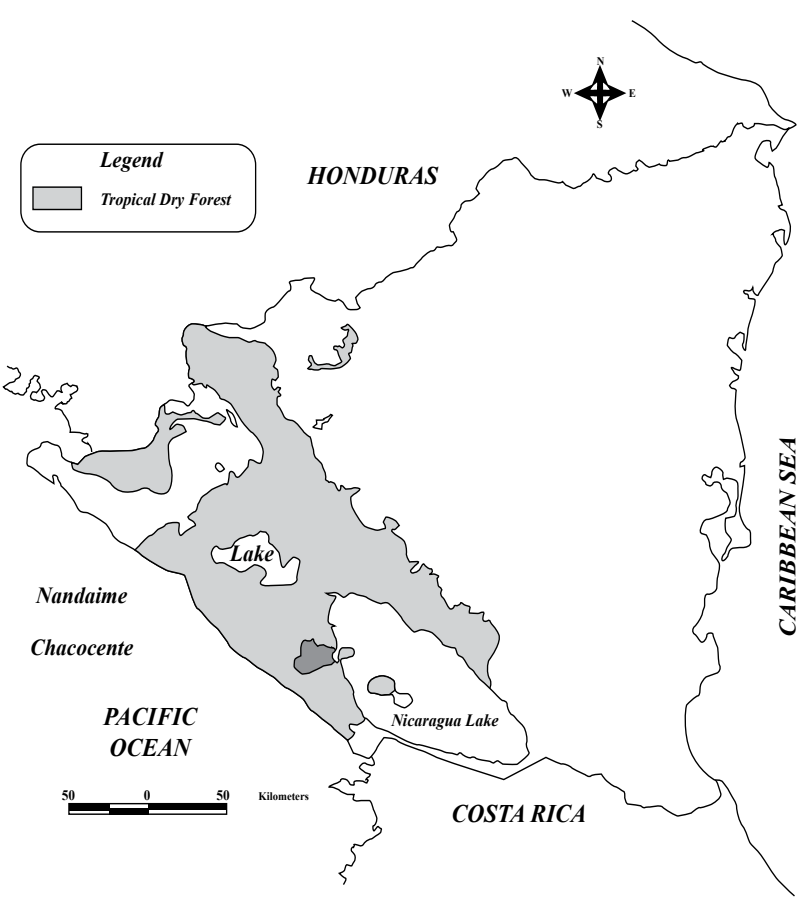

Figura 1. Distribución del Bosque Tropical Seco en Nicaragua. 


\section{Estudio del banco de semillas del suelo}

El banco de semillas del suelo es un componente importante para la regeneración del bosque tropical seco con este estudio tratamos de determinar la composición florística y la densidad de semillas presentes en el suelo.

Diseño de muestreo para el estudio de banco de semillas del suelo. Se utilizó un diseño de muestreo sistemático, el cual consistió en seleccionar de manera aleatoria un punto dentro del bosque y a partir de este, se estableció puntos de muestreo, con orientación Norte, Sur, Este y Oeste. La distancia entre puntos fue de 50 metros. Mediante el uso de un cuadrado de madera de $15 \mathrm{~cm}$ de largo y $15 \mathrm{~cm}$ de ancho, y $15 \mathrm{~cm}$ de altura se tomaba la muestra de suelo.

El muestreo en cada sitio incluía cuatro capas sucesivas de suelo separadas, que fueron removidas con la ayuda de un cuchillo filoso y cuchara de jardín, luego la muestra de cada capa se colocó en bolsas plásticas, marcadas con el nombre del sitio, número de línea, número de parcela y capa para llevarlas al invernadero Las muestras incluían la capa de litter y tres capas de suelo mineral sucesivas de tres centímetros de grosor (de 0-3 cms, de 3-6 cms y de 6-9 cms) (Teketay \& Granström, 1995). El número de muestras de suelo fueron 48 .

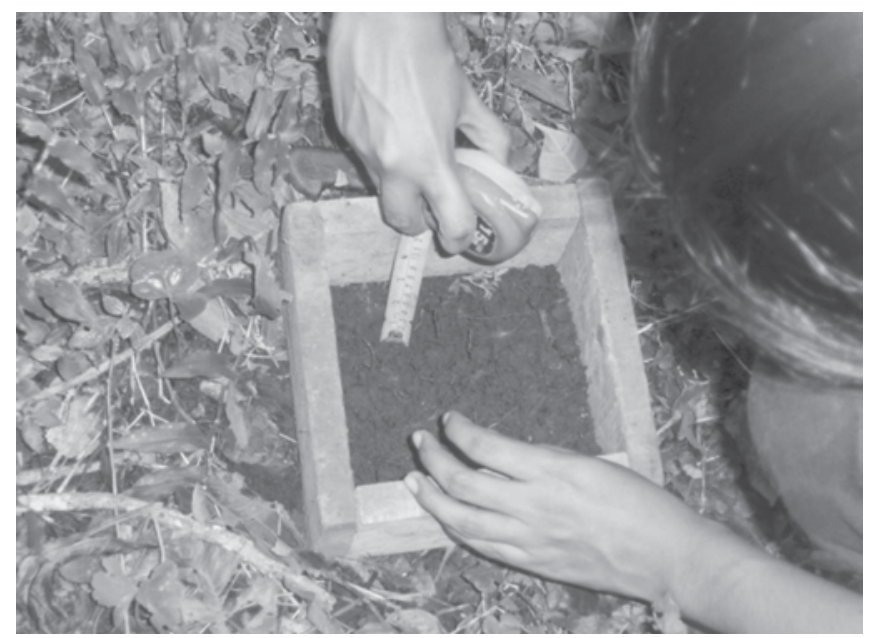

Figura 2. Cuadrado de madera de $15 \mathrm{~cm}$ de largo y $15 \mathrm{~cm}$ de ancho, y $15 \mathrm{~cm}$ de altura para la toma de la muestra de suelo.

Etapa de invernadero. Las muestras fueron trasladadas al invernadero de la Universidad Nacional Agraria en donde se colocaron en bandejas de plástico de $45 \mathrm{~cm} \mathrm{x}$ $25 \mathrm{~cm}$., distribuidas en tres bancales.

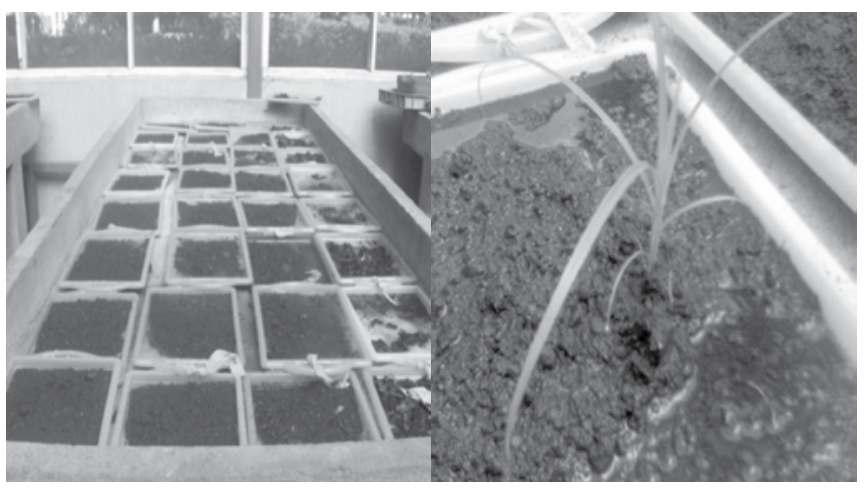

Figura 2. Cuadrado de madera de $15 \mathrm{~cm}$ de largo y $15 \mathrm{~cm}$ de ancho, y $15 \mathrm{~cm}$ de altura para la toma de la muestra de suelo.

Monitoreo de muestras. Las muestras se mantuvieron con riego periódico para proveer las condiciones ideales de germinación y se realizaron observaciones diariamente, durante tres meses, para determinar la emergencia total, la emergencia por especie.

Análisis de los datos. Se determinó la densidad de semillas por metro cuadrado, haciendo uso de la fórmula establecida para calcular número de árboles por hectárea (Sörgel, 1985), adaptada para determinar el número de semillas por metro cuadrado. A continuación se detalla el procedimiento:

No de sem $/ \mathrm{m}^{2}=\frac{1 \times \sum \mathrm{sem}}{(\mathrm{Nm} \times \mathrm{Ac})}$

Donde:

Nm: número de muestras

Ac: área del cuadrado utilizado $\left(0.0225 \mathrm{~m}^{2}\right)$

$\sum$ Sem: sumatoria de las semillas.

\section{Regeneración natural}

El estudio de regeneración natural se basa en determinar aspectos importantes de la regeneración tales como: abundancia, frecuencia y tasa Anual de cambio basado en un inventario forestal realizado en dos años consecutivos. De tal manera que los resultados obtenidos puedan contribuir al manejo del bosque seco en Nicaragua.

Especies seleciondas. $L$. minimiflorus, $T$. ochracea, $L$. divaricatum, fueron las especies seleccionadas para este estudio, basado en el interés de los productores en estas especies y también por el valor comercial.

Diseño del inventario para el estudio de la regeneración natural. El inventario utilizado en este estudio es un inventario sistemático en fajas continuas 
o líneas. Se estableció una línea base de inventario con azimut constante de $305^{\circ}$. Se establecieron cuatro líneas de inventario con espaciamiento entre línea de $800 \mathrm{~m}$. Cada línea tiene una longitud de $1000 \mathrm{~m}$ con $5 \mathrm{~m}$ de ancho y un azimut constante de $35^{\circ}$.

Disposición y tamaño de la parcela. Las parcelas se ubicaron en las líneas de inventario cada $100 \mathrm{~m}$; en un total de 10 parcelas por cada línea de inventario. $\mathrm{Su}$ forma es rectangular. Las dimensiones son las siguientes: 100 metros de largo por 5 metros de ancho, con lo cual se cubre un área de $500 \mathrm{~m} 2$ por cada una de las parcelas. Cada línea de inventario representa un área total de 0.5 ha. Lo cual representa 2 ha de área real muestreada. En la cual se recopiló información de la regeneración natural menor de $10 \mathrm{~cm}$ de DAP de las especies estudiadas.

\section{Estimación de abundancia, frecuencia absoluta y frecuencia relativa}

Abundancia: Es el parámetro que nos indica el número de arboles por especies. Está se divide en abundancia absoluta que es el número de individuo por especie y abundancia relativa la cual es la proporción de cada especie en el número total de individuos encontrados (Sáenz y Finegan, 2000).

Frecuencia Absoluta. Representa la proporción de las muestras ocupadas por los individuos de la especie expresada en porcentaje. Se calcula mediante la fórmula:

$$
\mathbf{F}=(\mathbf{M i}) \mathbf{x} \mathbf{M}
$$

Donde

F: Frecuencia

Mi: Número de unidades muestreales en la que aparecen los individuos.

M: Número total de unidades muestreales.

A través del cálculo de la frecuencia se puede tener una idea sobre la distribución que presenta la regeneración de una especie en particular (Matteucii, 1992). Encontramos cinco clases de frecuencia:

\begin{tabular}{cll}
\hline Clase de frecuencia & Rango $(\%)$ & Clasificación \\
\hline I & $1-20$ & Rara \\
II & $21-40$ & Ocasional \\
III & $41-60$ & Frecuente \\
IV & $61-80$ & Abundante \\
V & $81-100$ & Muy abundante \\
\hline
\end{tabular}

La abundancia y la frecuencia son dos parámetros muy importantes que permiten tener una visualización de cómo es el comportamiento de la dinámica del bosque con respecto a las especies estudiadas. La abundancia nos indica el potencial de la regeneración natural de determinada especie en función a la cantidad de individuos que se encuentran en área de muestreo.

Tasa anual de mortalidad (\%) (Primack et al., 1985)

Tam $=1-\left(\mathrm{N}_{\text {final }} / \mathrm{N}_{\text {inicial }}\right)^{1 / t}$

$\mathbf{N}_{\text {final }}=$ población al final del periodo

$\mathbf{N}_{\text {inicial }}=$ población al inicio del periodo

$\mathbf{f}=$ tiempo del periodo

\section{RESULTADOS Y DISCUSIÓN}

Composición y riqueza de especies del banco de semillas del suelo. El número total de especies presente en el banco de semillas del suelo fue de 33 especies, distribuidas en 23 familias. Es notable que la riqueza de especies y el número de familias aumenta a medida que la edad de la sucesión es mayor, resultados similares a los encontrados por Scholz et al (1999); según Lamprecht (1990), al aumentar la edad de la sucesión, el bosque presenta una mayor cantidad de especies, es más diverso, por lo que también se podría asumir que las probabilidades de que existan más especies, representadas por varias familias, almacenadas en el suelo sea mayor.

Tabla 1. Número de familias, géneros y especies por forma de vida en el banco de semillas del suelo en Nandarola, Nandaime, Nicaragua

\begin{tabular}{llccc}
\hline Sitio & $\begin{array}{c}\text { Forma de } \\
\text { vida }\end{array}$ & $\begin{array}{c}\mathrm{N}^{\circ} \mathrm{de} \\
\text { familias }\end{array}$ & $\begin{array}{c}\mathrm{N}^{\circ} \mathrm{de} \\
\text { géneros }\end{array}$ & $\begin{array}{c}\mathrm{N}^{\circ} \mathrm{de} \\
\text { especies }\end{array}$ \\
\hline La Chipota & Hierba & 9 & 11 & 13 \\
& Bejuco & 6 & 7 & 7 \\
& Arbustos & 4 & 4 & 4 \\
& Arboles & 8 & 9 & 9 \\
\hline Total & & 23 & 31 & 33 \\
\hline
\end{tabular}


Contrario con otros estudios que han reportado valores muy bajos de especies arbóreas almacenadas en el suelo (Gordón, 1999; Scholz et al, 1999; Teketay \& Granström, 1995), la proporción relativamente alta encontrada en este estudio de la fracción leñosa, puede relacionarse con la disponibilidad, dispersión y cercanía de la fuente de semillas (Gordón,1999), en especial árboles remanentes. Es posible que muchas semillas de comunidades adyacentes sean dispersadas por aire (anemócora).

Otros estudios reportan ausencia de semillas arbóreas en el banco de semillas de suelo por las siguientes causas: 1.- La probabilidad de que el muestreo se hiciese en un área con semillas arbóreas haya sido nula, 2. - las semillas desaparecieron del banco por germinación, descomposición o depredación, y 3. - el muestreo no coincidió con la época de fructificación y/o dispersión de la mayoría de árboles presentes.

En un estudio realizado en el Parque ecológico municipal, Canta-Gallo la herbácea anual Cyperus rotundus fue la especie dominante en el banco de semillas del suelo (White y Gámez 2009).

Densidad del banco de semillas del suelo. El número total de semillas presente fue 240 semillas por metro cuadrado. Que una cantidad relativamente baja. Estos resultados no concuerdan con los hallados por Scholz et al (1999), que encontraron cantidades mayores de semillas en un bosque seco secundario en Costa Rica. Los bancos de semillas del suelo contienen un alto número de semillas durante los primeros estadios de desarrollo o justo después del abandono del sitio por la actividad agrícola (Ashton et al, 1998), además las especies herbáceas producen una gran cantidad de semillas que se dispersan fácilmente y son las más abundantes en los bancos de semillas del suelo (Gordón, 1999).

La alta densidad de semillas de la familia Violaceae y Poaceae, se debió a que estuvieron representadas por especies que en total reportaron la mayor cantidad de semillas (562 semillas), como: Hybanthus attenuates (garbaceo), Cenchrus echinatus (mozote de caballo), Sorghun halapensis (zacate invasor), Cynodon dactylon (zacate gallina), Paspalum sp. (zacate peludo), Cenchrus undatus (mozote de perro) y Boutelowa alamosana (guineíta) pertenecientes a la forma de vida herbácea; estas especies producen semillas pequeñas en gran cantidad que se dispersan fácilmente por aire, son especies agresivas que colonizan fácilmente áreas nuevas y una vez establecidas almacenan grandes cantidades de semillas en el suelo (Scholz et al, 1999).

\section{Regeneración natural}

Abundancia y frecuencia de la regeneración natural de las cuatro especies forestales. La abundancia y la frecuencia son dos parámetros muy importantes que permiten tener una visualización de cómo es el comportamiento de la dinámica del bosque con respecto a las especies estudiadas. La abundancia nos indica el potencial de la regeneración natural de determinada especie en función a la cantidad de individuos que se encuentran en área de muestreo.

Tabla 2. Abundancia y frecuencia de la regeneración natural de cuatro especies en el bosque seco tropical de Chococente, Carazo

\begin{tabular}{|c|c|c|c|c|c|}
\hline Especie & $\begin{array}{c}\text { Abundancia } \\
\text { Absoluta } \\
\text { (ind/ha) }\end{array}$ & $\begin{array}{c}\text { Abundancia } \\
\text { Relativa } \\
(\%)\end{array}$ & $\begin{array}{c}\text { Frecuencia } \\
\text { Absoluta } \\
(\%)\end{array}$ & $\begin{array}{l}\text { Clases de } \\
\text { Frecuencia }\end{array}$ & Clasificación \\
\hline neochrysantha (c) & 91.5 & 55.0 & 65.0 & IV & Abundancia \\
\hline I. americanus $(t)$ & 49.0 & 29.0 & 32.5 & II & Ocasional \\
\hline . candidissimum (m) & 23.0 & 14.0 & 22.5 & II & Ocasional \\
\hline divaricatum (Q) & 4.0 & 0.2 & 17.5 & I & Rara \\
\hline otal & 167.5 & 100 & & & \\
\hline
\end{tabular}
distribución espacial de la regeneración natural de las especies en estudio.

Tasa anual de cambio en densidad de la vegetación. La tasa anual de cambio varió considerablemente en cada tamaño de la vegetación de las especies estudiadas. La densidad de brinzales de $L$. minimiflorus disminuyo por $54 \%$, mientras que la densidad de latizales incremento $2 \%$. Para T. ochracea, la densidad de brinzales,

Tabla 3. Tasa Anual de cambio en densidad de brinzales, latizales y fustales y densidad total de Lonchocarpus minimiflorus, Tabebuia ochracea and Lysiloma divaricatum en un periodo de dos años en el Bosque tropical Seco de Chacocente

\begin{tabular}{lccc}
\hline Tamaño & \multicolumn{3}{c}{ Tasa anual de cambio } \\
\hline & L. minimiflorus & T. ochracea & L. divaricatum \\
\hline Brinzales & -0.544 & 0.176 & 0.112 \\
Latizales & 0.017 & 0.004 & -0.693 \\
Fustales & ---- & 0.458 & ----- \\
\hline Total & -0.129 & 0.052 & -0.144 \\
\hline
\end{tabular}


latizales y fustales incremento por $18 \%, 0.4 \%$ y $46 \%$ respectivamente.

La especie $L$. divaricatum, tuvo $69 \%$ de pérdidas de latizales comparado con un pequeño incremento en la densidad de brinzales (11\%). De manera general la densidad fue ligeramente positiva $(5 \%)$ para $T$. ochracea.

\section{Implicaciones para el manejo del bosque seco tropical}

La mayoría de las especies del banco de semillas del suelo del bosque seco son hierbas, lo que implica que la regeneración de árboles del bosque seco sería difícil y lenta después de un disturbio, al haber poca reserva de semillas de especies arbóreas en el suelo. La presencia de semillas de especies arbóreas fue muy baja, por lo cual se recomienda aplicar tratamientos silviculturales tal como plantaciones de enriquecimiento con especies seleccionadas por los mismos habitantes de la zona que permita mejorar la composición florística de la vegetación.

Una de las formas más económicas y prácticas de restauración es la regeneración natural, donde la única tarea es aislar el área que se desea restaurar de cualquier fuente de perturbación, para que los procesos naturales de la sucesión sigan actuando, recuperando la condición de bosque maduro. Sin embargo, debido a la baja persistencia de las semillas de los bosques tropicales secos e $\mathrm{n}$ el banco de semillas, la regeneración natural podría estar totalmente comprometida una vez que no existieran fuentes de propágulos muy cercanas (un fragmento grande por ejemplo), que pudieran funcionar como fuente de semillas. Por lo tanto, en los bosques tropicales secos, la regeneración natural como alternativa de restauración presenta mucho más restricciones

Es conveniente introducir plántulas relativamente grandes en el campo, para que cuando llegue la época de sequía ellas posean un sistema radicular bien desarrollado y puedan soportar los meses de sequía.

En conclusión podemos afirmar de acuerdo a los resultados obtenidos que la regeneración natural no es suficiente 'para restaurar las especies estudiadas, especialmente: L. divaricatum y L. minimiflorus cuyas densidad demostró una tendencia a disminuir. Por lotanto, se deben de tomar algunas medidas para incrementar la regeneración natural en áreas abiertas. Tratamientos silviculturales tales como enriquecimiento se deben de considerar para futuros trabajos de restauración.

\section{REFERENCIAS BIBLIOGRÁFICAS}

Ashton, PMS; Harris, PG; Thadani, R. 1998. Soil seed bank dynamics in relation to topographic position of a mixed - deciduos forest southern New England, USA. Forest Ecology and Management 111:15 - 22.

Bazzaz, FA; Pickett, STA. 1980. Physiological ecology of tropical succession: a comparative review. Ann. Rev. Ecol. Syst. 11: 287-310.

Brown S; Lugo AE. 1990. Tropical secondary forest. Journal of Tropical Ecology, 6: 1-32.

Gordón, E. 1999. Dinámica de la vegetación y del banco de semillas en un humeda herbáceo lacustrino (en línea). Caracas, Venezuela. Consultado 10 Feb. 2005. Disponible en: http://rbt.ots.ac.cr/revistas/ butgordon.htm.

Janzen, DH. 1986. Tropical dry forest: the most endangered major tropical ecosystem, p. 130-137. In E.O. Wilson (ed.). Biodiversity. National Academic, Washington DC, EEUU.

Janzen, DH. 2000. Costa Ricas Área de Conservación Guanacaste: a long march to survival through nondamaging biodevelopment. Biodiversity 1: 7-20.

Kalacska, M; Sanchez-Azofeifa, GA; Calvo-Alvarado, JC; Quesada M, RB; Janzen, DH. 2004. Species composition, similarity and diversity in three successional stages of a seasonally dry tropical forest. Forest Ecology and Management, 200: 227-247.

Teketay, D. 1997. Seedling population and regeneration of woody species en dry Afromontane forests of Ethiopia. Forest Ecology and Management, 98: 149 - 165 
Teketay, D; Granström, A. 1995. Soil seed banks in dry afromontane forest of Etiopía. Journal of Vegetation. Science 6: $777-786$

Tekle, K; Bekele, T. 2000. The role of soil seed banks in the rehabilitation of degraded hill slopes in Southern Wello, Ethiopia. Biotropica, 32: 23-32.

Kramer, EA. 1997. Measuring landscape changes in remnant tropical dry forest, p.386-399. In W.F. Lawrence \& R.G. Bierregaard (eds.). Tropical forest remnants: ecology, management, and conservation of fragmented communities. Chicago University, Chicago, EEUU.

Lamprecth, H. 1990. Silvicultura en los trópicos. Edición en español. 335 p.

Matteucci, S. Colma. 1992. Metodología para el estudio de la vegetación. Secretaria general de la organización de los estados americanos. Programa regional de desarrollo científico y tecnológico. Washington monografía $\mathrm{N}^{\circ} 22$

Mulkey, SS; Chazdon, RL; Smith, AP. 1996. Tropical forest plant ecophysiology. Chapman \& Hall, Nueva York, EEUU.

Primack, RB; Ashton, PS; Chai, P; Lee, HS. 1985 Growth rates and population structure of Moraceae trees in Sarawak. Ecology 66: 577- 588

Scholz, C; Gonzáles, E; Vilchez, B. 1999. El Banco de Semillas en Diferentes Estados Sucesionales en un Bosque Seco Tropical de Costa Rica. Tesis Ing. Forestal. San Pedro, Costa Rica. OTS (Organization for tropical studies).78p.

Saenz, G; Finegan, B. 2000. Monitoreo de la regeneración natural con fines de manejo forestal. $\mathrm{N}^{\circ} 15$. CATIE, Turrialba, Costa Rica.

White, E; Gamez-López, J. Evaluación del banco de semillas del suelo en tres ecosistemasdel Parque Ecológico Municipal Canta Gallo, Condega, Nicaragua

Zimmerman, JK; Pascarella, JB; Aide, TM. 2000. Barriers to forest regeneration in an abandoned pasture in Puerto Rico. Restoration Ecology, 8: 350-360. 